\title{
The Overall Design of Science and Technology Management Information System based on B/S Structure
}

\author{
Xiangling Lei ${ }^{1,2, a}$, Linying $X u^{1, b}$ \\ 1 TianjinUniversity, Tianjin, Tianjin, China \\ 2 XiZang Agriculture and Animal Husbandry College, Linzhi, Tibet, China \\ a303434201@qq.com, b591517678@qq.com
}

\begin{abstract}
Keywords: Science and Technology Management, Information System, MIS System.
Abstract: The revolution of computer and network technology forcing the science and technology departments to realize the science, standardization, modernization of science and technology management. According to MIS system model, this paper did the overall design of science and technology management information system based on $\mathrm{B} / \mathrm{S}$ structure, aiming to clarify the main functions of each layer of the model, including system requirement analysis, key module design, and database design for MIS, that lay the foundation for the realization of science and technology management information system.
\end{abstract}

\section{Introduction}

Revolution of computer and network technology provides sufficient condition for collection, transmission and sharing of information which are included in informatization, and today, informatization has become major trends of global economic and social development. As the representative of the high and new technology, information technology has increasingly becoming an important productive force to promote the development of human society.

In work practice, in order to fully realize science, standardization and modernization of management, science and technology departments urgent need using modern electronic technology and information technology, and take the network of information transmission, the business process of electronic as a means. To this end, fully using the advantages of networks, setting up a set of systems of scientific, perfect and efficient information management, exchange, release, storage, providing quick and effective means to planning management and statistics, and providing effective support to science and technology manage decisions.

\section{System Requirement Analysis}

Through the establishment of this system, a unified and efficient information network will be formed in science and technology department, so it can realize gradually summary to online research statistics information timely and accurately, realize query of online science information top down and automatically generate various types of scientific research and statistical reports, and realize daily information management of science work. It is specific performance as follows:

1) System uses data dictionary as drive, and make it meet requires of science management business.

2) Flexible and convenient statistical construction tool.

3) Powerful report making tool, which can automatically generate annual statistics of scientific research.

4) Full realization and data sharing of other scientific research management system.

5) Support multi user, strong security.

\section{Key Module Design}

The main task of the system is to focus on the document data obtained from the survey, analysis of the internal management of the organization and information processing process. According to the 
functional organization of system, system is divided into two parts: static information announcement and dynamic processing.

Static Information Announcement. The content of static is mainly Information delivery, include latest scientific research, scientific and educational information at home and abroad, enterprises need information and relevant research management regulations. Scientific research departments need to browse this page regularly every day, then inform the relevant personnel of the latest information, other people can also surf these information on the Internet.

Dynamic Processing. The content of dynamic is mainly business process. The management statistics and analysis of various data include generation and download of statistical report, generation of query statistics, data entry management and system rights management.

Structure Design of Modular. Science and technology information management system include four parts: comprehensive query (Project declaration, rewarding), comprehensive statistics (Project declaration, rewarding), data dictionaries (Technical title, education degree) and system management (data backup, data recovery).

\section{Database Design for MIS System}

From the view of software life cycle, the whole process of data management design can be divided into four stages [1]: analyzing users' requirements; trying to design; conceptual model and external model design; physical design.

The first stage: collection and analysis of user requirements, including data requirements, processing requirements and restrictions, etc.

The second stage: express users' data, involving logical data model.

The third and forth stage: concretely realize data management.

The principle of database system code is:

The importance of MIS system data platform. At present, the vast majority of MIS systems are man-machine systems which based on the database. The role of MIS is can not play out through a development work, but the repeated actual work. It is wrong to think that the MIS system can be used for several decades after the completion. Only meet the current functionality, the system is generally difficult to run a long time. Therefore, the development of the system should be able to facilitate the adjustment and expansion [2].

American scholar Martin pointed out: the processing process enterprise data is changeable, but the basic data is stable and unchanging. Whatever changes of management model, the basic data types are stable and unchanging. As long as the underlying data is stable, and make data platform upgraded with database, the system could be long term used. Therefore, data platform is the foundation of the system construction and the core of the MIS system, and also the essence of the construction of MIS [3].

Function design of database system. To establish a data platform is to organize the data into the database, so the design of the database and the establishment of the data platform is very important.

1) The functional characteristics of database system [4]

a. The independence of data: the data is independent of the application;

b. The security of data: irrelevant personnel can not get data that he should not get.

c. The integrity of data: data should be correct, true and objectively, it is divided into logical integrity, operational integrity and physical integrity.

Logical integrity means that any changes in the database should reflect the true state of the objective world. Operational integrity is that preventing database damage through reasonable concurrency control when multiple users at the same time. The physical integrity is the correctness of the physical data

d. The uniformity of data: data should be compatible.

e. Data sharing: it is one of the characteristics of the database system, embodied in follows: 
The multiple applications can use the same database; multiple users can access the same data at the same time; the new software can be increased without increasing new storage space; it can be opened to society, and become a kind of social information resources.

f. Control redundancy: Control redundancy is necessary to large database, especially in distributed environment, it can reduce the time overhead of data transmission on the network. But it needs to be controlled within a proper ranges, this can save space and reduce the database overhead, preventing data inconsistency

g. Centralized management: Overall manage the operations of data reading and writing, class size, size, security and confidentiality checks and others.

h. Concurrency control: Concurrency control is control strategy that multiple modified operations to database at the same time, the commonly used method is to add lock. The locking overhead is smaller with unit of the lock is greater, but the degree of parallelism is lower.

i. Failure recovery: restore database system to correct state after the database had been destroyed. The recovery strategy is often used to establish a copy, log, establish a checkpoint, a differential file and a transaction commit.

g. Data dictionary: it holds the information without in the database, such as patterns, associations, types of data items, length, user ID, password, index, etc.

2) Selection of database server

This system uses SQL Server 2000 database which is developed by Microsoft Corp. SQL Server 2000 has almost all the advantages of the development of Web database system. It has Powerful function, strong system security, and the operation is relatively simple compared to other database, and because it is a product of Microsoft Corp, the Server operating system between SQL Server 2000 and WINDOWS system uses underlying technology with seamless combination, so SQL Server 2000 has a higher security mechanism [5]. Therefore, using Server ASP+SQL to develop Web application can achieve rapid interaction between the user and the database.

3) The standardization of database storage structure design

MIS system is an application system based on database. Reasonable database design plays an important role in MIS system.

Although the demand analysis is good, but if the database design is reasonable, not only can make the program design work get half the results with double the effort, but also cause the whole system is full of mistake in the practical applications in the future, thus greatly increase the difficulty of maintenance and input, and even lead to paralysis of the entire system.

At the stage of demand analysis, regardless of the data flow diagram obtained by the structural analysis, or data class from object-oriented analysis and identification, Are not wholly intact directly into the database table, which must be through standardization. Standardization is that the data storage structure should meet certain restrictions. These restrictions can be divided into several levels in accordance with the strict degree, respectively are the first normal form and the second normal form, in turn, there are third normal form, forth normal form and fifth normal form. According to the characteristics and practical experience of MIS system, the actual needs of MIS system can be basically meet if the standardization of database system storage structure to the third normal form [6].

Specifically speaking, it meets the first normal form after eliminating data redundancy of normalized data; it meets the second normal form after eliminating functional dependency of the data storage structure; it meets the third normal form after eliminating transfer functional dependency.

With standardized methods, we also need to know the object of standardization. Basic data classes should form basic tables. Derived data class should form temporary tables, or define as views.

\section{Conclusions}

According to MIS system model, this paper did the overall design of science and technology management information system based on B/S structure, aiming to clarify the main functions of each layer of the model, including system requirement analysis, key module design, and database design for 
MIS, that lay the foundation for the realization of science and technology management information system.

\section{References}

[1] Xiaojun He, Xujing Cui. Integration and design of WWW database information service system: Journal of Shenyang Institute of Technology Vol. 19 (2014), p.18. In Chinese

[2] Zhigang Wu, Binxing Fang. Information resource publishing model based on WEB: Computer Application Research Vol. 102 (2012), p.21-23. In Chinese

[3] Hua Yu. Construction of management information system based on INTERANET: Management Information Systems Vol. 27 (2009), p.57-60. In Chinese

[4] Zheng Li. Analysis and application of WEB and database integration technology: Modern Computer Vol. 55 (2014), p.41-43. In Chinese

[5] Guangqi Huang: WEB database Interconnection Technology Development Guide (National Defence Industry Press, China 2000). In Chinese

[6] Ping Li, Bin Liu. WEB database interface technology and its application: Application of computer system Vol. 64 (2011), p.26-29. In Chinese 